\title{
Study of baryonic decays of $B$ mesons at BABAR
}

\section{Oliver Gruenberg* (for the BABAR collaboration)}

Rostock University

E-mail: oliver.gruenberg@uni-rostock.de

\section{$B A B A R$}

We report on recent $B A B A R$ analyses of the baryonic $B$ decays $\bar{B}^{0} \rightarrow \Lambda_{\mathrm{c}}^{+} \bar{p} \pi^{+} \pi^{-}, B^{-} \rightarrow$ $\Sigma_{c}^{++} \bar{p} \pi^{-} \pi^{-}, \bar{B}^{0} \rightarrow \Lambda_{\mathrm{c}}^{+} \bar{p} p \bar{p}$ and $\bar{B}^{0} \rightarrow D^{0} \Lambda \bar{\Lambda}$. The used data sample contains $471 \times 10^{6} B \bar{B}$ pairs that were generated in the process $e^{+} e^{-} \rightarrow \Upsilon(4 S) \rightarrow B \bar{B}$ and collected with the BABAR detector at the PEP-II storage ring at SLAC. We find $\mathscr{B}\left(\bar{B}^{0} \rightarrow \Lambda_{\mathrm{c}}^{+} \bar{p} \pi^{+} \pi^{-}\right)=\left(12.3 \pm 0.5_{\text {stat }} \pm 0.7_{\text {syst }} \pm\right.$ $\left.3.2_{\Lambda_{\mathrm{c}}}\right) \times 10^{-3}, \mathscr{B}\left(B^{-} \rightarrow \Sigma_{c}^{++} \bar{p} \pi^{-} \pi^{-}\right)=\left(2.98 \pm 0.16_{\text {stat }} \pm 0.15_{\text {syst }} \pm 0.77_{\Lambda_{\mathrm{c}}}\right) \times 10^{-3}$, where the last uncertainty is due to $\mathscr{B}\left(\Lambda_{\mathrm{c}}^{+} \rightarrow p K^{-} \pi^{+}\right)$, respectively. For the decay $\bar{B}^{0} \rightarrow \Lambda_{\mathrm{c}}^{+} \bar{p} p \bar{p}$ we see no events and set an upper limit $\mathscr{B}\left(\bar{B}^{0} \rightarrow \Lambda_{\mathrm{c}}^{+} \bar{p} p \bar{p}\right) \times \frac{\mathscr{B}\left(\Lambda_{\mathrm{c}}^{+} \rightarrow p K^{-} \pi^{+}\right)}{0.050}<2.8 \times 10^{-6}$ at $90 \% \mathrm{CL}$, where we have normalized $\mathscr{B}\left(\Lambda_{\mathrm{c}}^{+} \rightarrow p K^{-} \pi^{+}\right)$to the world average value. There is evidence for the decay $\bar{B}^{0} \rightarrow D^{0} \Lambda \bar{\Lambda}$ and we measure $\mathscr{B}\left(\bar{B}^{0} \rightarrow D^{0} \Lambda \bar{\Lambda}\right)=\left(9.8_{-2.6}^{+2.9}\right.$ stat $\left.\pm 1.9_{\text {syst }}\right) \times 10^{-6}$ corresponding to a significance of $3.4 \sigma$.

XV International Conference on Hadron Spectroscopy 4-8/11/2013

Nara, Japan

${ }^{*}$ Speaker. 


\section{Introduction}

From ARGUS measurements published in 1992 it is known that approximately $7 \%$ of all $B$ decays have baryons among their final states [1]. This is a substantial fraction which has motivated a large number of studies on exclusive decay modes that were published in the last twenty years, mainly by BABAR, Belle, and CLEO. However, until today the branching fractions of these modes sum up to only $(0.53 \pm 0.06) \%$ for $B^{0}$ and $(0.85 \pm 0.15) \%$ for $B^{+}$[2], and leaving us the until now unsolved $B \rightarrow$ baryon puzzle.

Due to its large mass, $B$ mesons are able to decay to a large spectrum of baryons with different flavours such as $p, \Lambda, \Lambda_{\mathrm{c}}^{+}, \Xi_{c s}$, as well as their isospin partners and excited states. Hence exclusive modes of baryonic $B$ decays are a good place to search for exotic baryons. On the other hand baryonic $B$ decays allow better understanding of hadronization into baryons at low $q^{2}$ values. One feature that has been observed in a number of decay channels is an enhanced baryon production at the baryon-antibaryon mass threshold and the dependence of the branching fraction on the multiplicity; it can be stated that $\mathscr{B}(B \rightarrow N \bar{N})<\mathscr{B}(B \rightarrow N \bar{N} \pi)<\mathscr{B}(B \rightarrow N \bar{N} \pi \pi)$. In addition, it has been found that baryonic $B$ decays may have complex resonant substructures and that the branching fraction of decays of the sort $B \rightarrow \Lambda_{\mathrm{c}}^{+} \bar{p}+n \cdot \pi$ with $n=1,2,3$ have large contributions from intermediate states including baryon resonances.

In the following we present recent $B A B A R$ results on studies of the baryonic $B$ decays ${ }^{1} \bar{B}^{0} \rightarrow$ $\Lambda_{\mathrm{c}}^{+} \bar{p} \pi^{+} \pi^{-}, \bar{B}^{0} \rightarrow \Lambda_{\mathrm{c}}^{+} \bar{p} p \bar{p}, B^{-} \rightarrow \Sigma_{c}^{++} \bar{p} \pi^{-} \pi^{-}$, and $\bar{B}^{0} \rightarrow D^{0} \Lambda \bar{\Lambda}$ using the full BABAR dataset with an integrated luminosity of $426 \mathrm{fb}^{-1}$, which corresponds to $471 \times 10^{6} B \bar{B}$ pairs.

\section{2. $\bar{B}^{0} \rightarrow \Lambda_{\mathrm{c}}^{+} \bar{p} \pi^{+} \pi^{-}[3]$}

In the analysis the total signal yield is extracted in the plane of the reconstructed $B$ mass $m_{\text {inv }}$ and $m\left(\Lambda_{\mathrm{c}}^{+} \pi\right)$ (Fig. 1) to seperate contributions that include intermediate states with $\Sigma_{\mathrm{c}}(2455,2520)^{0,++} \rightarrow \Lambda_{\mathrm{c}}^{+} \pi^{-,+}$(four exclusive modes) from the remaining non- $\Sigma_{\mathrm{c}}$ signal. It shows that decay modes with a $\Sigma_{c}^{++}$are prefered w.r.t. $\Sigma_{c}^{0}$. Summing up, the four resonant three-body modes amount to about one third of the total branching fraction. The measured branching fraction is $\mathscr{B}\left(\bar{B}^{0} \rightarrow \Lambda_{\mathrm{c}}^{+} \bar{p} \pi^{+} \pi^{-}\right)=$ $\left(12.3 \pm 0.5_{\text {stat }} \pm 0.7_{\text {syst }} \pm 3.2_{\Lambda_{\mathrm{c}}}\right) \times 10^{-3}$

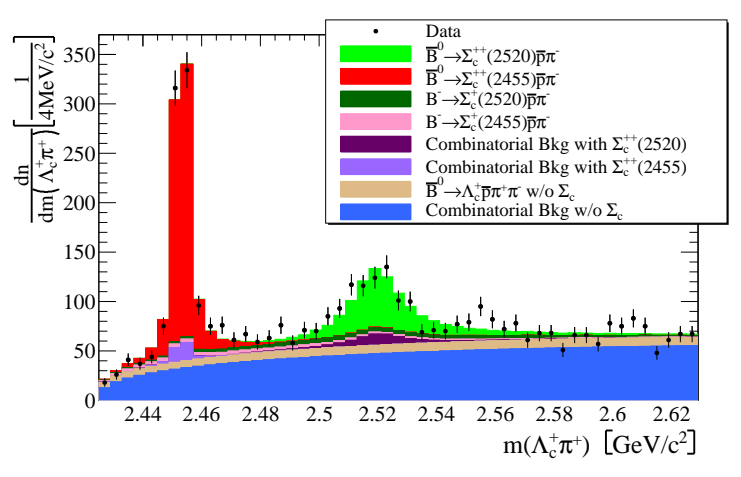

Figure 1: The $m\left(\Lambda_{\mathrm{c}}^{+} \pi^{+}\right)$distribution in data (points with errors) and the different signal and background contributions fitted to the data.

Beside the $\Sigma_{\mathrm{c}}(2455)$ and $\Sigma_{\mathrm{c}}(2520)$ resonances that are accounted for in the analysis, other intermediate states are visible in the invariant mass spectra of $\Lambda_{\mathrm{c}}^{+} \pi, \bar{p} \pi$ and $\pi^{+} \pi^{-}$. There are suggestions for the $\Sigma_{\mathrm{c}}(2800), \bar{\Delta}^{--}$and $\rho(770)$ [3]. Similar to the situation of $\Sigma_{\mathrm{c}}(2455)$ and $\Sigma_{\mathrm{c}}(2520)$, the signal for the $\Sigma_{\mathrm{c}}(2800)^{++}$is more pronounced than for the $\Sigma_{\mathrm{c}}(2800)^{0}$.

\footnotetext{
${ }^{1}$ The use of charge conjugate decays is implied throughout this text.
} 


\section{3. $\bar{B}^{0} \rightarrow \Lambda_{\mathrm{c}}^{+} \bar{p} p \bar{p}[4]$}

This decay is interesting in comparison to $\bar{B}^{0} \rightarrow$ $\Lambda_{\mathrm{c}}^{+} \bar{p} \pi^{+} \pi^{-}$because $\bar{B}^{0} \rightarrow \Lambda_{\mathrm{c}}^{+} \bar{p} p \bar{p}$ cannot have intermediate states, for example due to $\Sigma_{\mathrm{c}}$ or $\bar{\Delta}$ resonances, and has a very small phase space

$$
\frac{\int d \mathrm{PS}\left(\bar{B}^{0} \rightarrow \Lambda_{\mathrm{c}}^{+} \bar{p} p \bar{p}\right)}{\int d \operatorname{PS}\left(\bar{B}^{0} \rightarrow \Lambda_{\mathrm{c}}^{+} \bar{p} \pi^{+} \pi^{-}\right)} \approx \frac{1}{1400} .
$$

The ratio of $\mathscr{B}\left(\bar{B}^{0} \rightarrow \Lambda_{\mathrm{c}}^{+} \bar{p} p \bar{p}\right)$ over $\mathscr{B}\left(\bar{B}^{0} \rightarrow\right.$ $\Lambda_{\mathrm{c}}^{+} \bar{p} \pi^{+} \pi^{-}$) may be larger than the phase space factor of $1 / 1400$ due to the the fact that the signal of $\bar{B}^{0} \rightarrow \Lambda_{\mathrm{c}}^{+} \bar{p} p \bar{p}$ is limited to a small phase space and the invariant masses of $\Lambda_{\mathrm{c}}^{+} \bar{p}$ and $p \bar{p}$ are generally low and hence the hadronization into baryons may be ruled by threshold enhancement effects.

In our analysis we define a signal region in the plane of the reconstructed $B$ mass $m_{\mathrm{B}}$ and the energy-substituted mass $m_{\mathrm{ES}} \approx$ $\sqrt{(\sqrt{s} / 2)^{2}-p_{\mathrm{B}}^{2}}$, with $\sqrt{s}$ the center-of-mass energy (the exact formula takes into account the boost of the initial $e^{+} e^{-}$system in the laboratory frame). The signal region is an ellipse that uses the mean and the standard deviation of $m_{\mathrm{ES}}$ and $m_{\mathrm{B}}$ extracted from fits to the signal MC sample (Fig. 2).

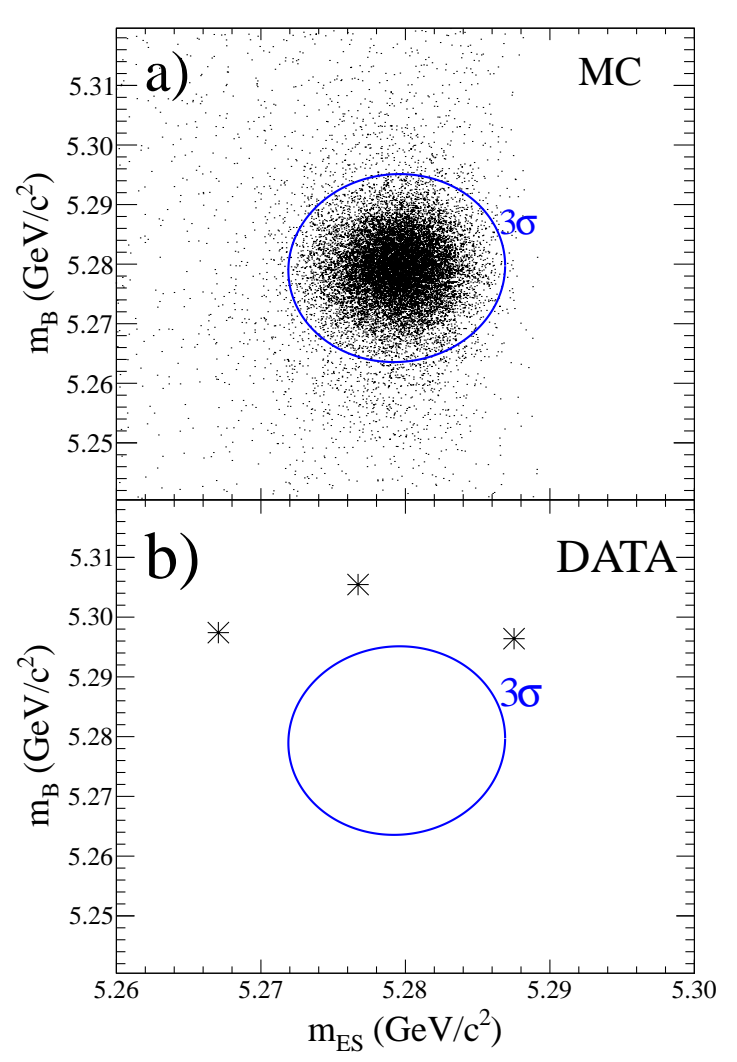

Figure 2: The $m_{\mathrm{ES}}$ vs. $m_{\mathrm{B}}$ distribution of selected events in (a) signal MC and (b) data. No signal candidates are observed within the signal region of the data sample.

We find no events in the signal region in the BABAR data and set an upper limit on the branching fraction

$$
\mathscr{B}\left(\bar{B}^{0} \rightarrow \Lambda_{\mathrm{c}}^{+} \bar{p} p \bar{p}\right) \times \frac{\mathscr{B}\left(\Lambda_{\mathrm{c}}^{+} \rightarrow p K^{-} \pi^{+}\right)}{0.050}<2.8 \times 10^{-6} \text { at } 90 \% \mathrm{CL}
$$

where we have normalized $\mathscr{B}\left(\Lambda_{\mathrm{c}}^{+} \rightarrow p K^{-} \pi^{+}\right)$to the world average value [2]. We compare this upper limit to $\mathscr{B}\left(\bar{B}^{0} \rightarrow \Lambda_{\mathrm{c}}^{+} \bar{p} \pi^{+} \pi^{-}\right)_{\text {non-res }} \approx 50 \% \cdot \mathscr{B}\left(\bar{B}^{0} \rightarrow \Lambda_{\mathrm{c}}^{+} \bar{p} \pi^{+} \pi^{-}\right)=6 \cdot 10^{-4}$, which is $\mathscr{B}\left(\bar{B}^{0} \rightarrow \Lambda_{\mathrm{c}}^{+} \bar{p} \pi^{+} \pi^{-}\right)$without contributions from intermediate states with $\Sigma_{\mathrm{c}}(2455,2520)^{0,++} \rightarrow$ $\Lambda_{\mathrm{c}}^{+} \pi^{-,+}$and estimated contributions from intermediate states with $\bar{\Delta}, N^{*}$ and $\rho$ resonances that are visible in the spectra of $m\left(\bar{p} \pi^{ \pm}\right)$and $m\left(\pi^{+} \pi^{-}\right)$. We find that there is no significantly enhanced hadronization into baryons due to threshold enhancement effects in the small phase space of the decay. This can be quantified by an effective enhancement factor that is smaller than seven.

$$
\frac{\mathscr{B}\left(\bar{B}^{0} \rightarrow \Lambda_{\mathrm{c}}^{+} \bar{p} p \bar{p}\right)}{\mathscr{B}\left(\bar{B}^{0} \rightarrow \Lambda_{\mathrm{c}}^{+} \bar{p} \pi^{+} \pi^{-}\right)_{\text {non-res }}} \lesssim 7 \times \frac{1}{1400}
$$




\section{4. $B^{-} \rightarrow \Sigma_{c}^{++} \bar{p} \pi^{-} \pi^{-}[5]$}

This decay is a resonant subchannel of $B^{-} \rightarrow$ $\Lambda_{\mathrm{c}}^{+} \bar{p} \pi^{+} \pi^{-} \pi^{-}$, which has the largest known branching fraction among all baryonic $B$ decays, and its studies may allow better understanding of the resonant substructure of baryon production in $B$ decays. The $\Sigma_{c}^{++}$is reconstructed as $\Sigma_{c}^{++} \rightarrow\left(p K^{-} \pi^{+}\right)_{\Lambda_{\mathrm{c}}} \pi^{+}$. In the $B A B A R$ data we find $787 \pm 43$ signal events with a reconstruction efficiency of $11.3 \%$ determined from signal MC. Therefore we derive $\mathscr{B}\left(B^{-} \rightarrow \Sigma_{c}^{++} \bar{p} \pi^{-} \pi^{-}\right)=\left(2.98 \pm 0.16_{\text {stat }} \pm\right.$ $\left.0.15_{\text {syst }} \pm 0.77_{\Lambda_{\mathrm{c}}}\right) \cdot 10^{-4}$ which is about $14 \%$ of $\mathscr{B}\left(B^{-} \rightarrow \Lambda_{\mathrm{c}}^{+} \bar{p} \pi^{+} \pi^{-} \pi^{-}\right)$. There is a suggestion for $\Lambda_{\mathrm{c}}(2595)^{+}$but not for other known $\Lambda_{c}^{*+}$ in the invariant mass spectrum of $\Sigma_{c}^{++} \pi^{-}$. We see unexplained structures in the spectrum of $m\left(\Sigma_{c}^{++} \pi^{-} \pi^{-}\right)$(Fig. 3).

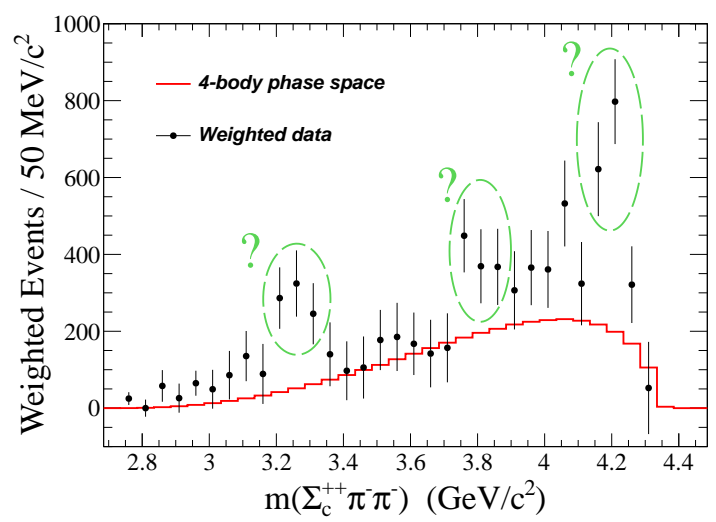

Figure 3: The $m\left(\Sigma_{c}^{++} \pi^{-} \pi^{-}\right)$distribution in data after efficiency correction and $\Delta E$ sideband subtraction compared to simulated four-body phase space decays.

\section{Threshold enhancement}

The threshold enhancement has been observed in a number of baryonic $B$ decays and may be a key feature in understanding hadronization processes into baryons. Figure 4 shows the invariant baryon-antibaryon mass of the two resonant subchannels $\bar{B}^{0} \rightarrow \Sigma_{c}^{++}(2455) \bar{p} \pi^{-}$and $\bar{B}^{0} \rightarrow$ $\Sigma_{c}^{0}(2455) \bar{p} \pi^{+}$of the decay $\bar{B}^{0} \rightarrow \Lambda_{c}^{+} \bar{p} \pi^{+} \pi^{-}$. It can be seen that in the data sample the decay rate of $\bar{B}^{0} \rightarrow \Sigma_{c}^{++}(2455) \bar{p} \pi^{-}$is clearly enhanced at the mass threshold in contrast to the distribution of $\bar{B}^{0} \rightarrow \Sigma_{c}^{0}(2455) \bar{p} \pi^{+}$.

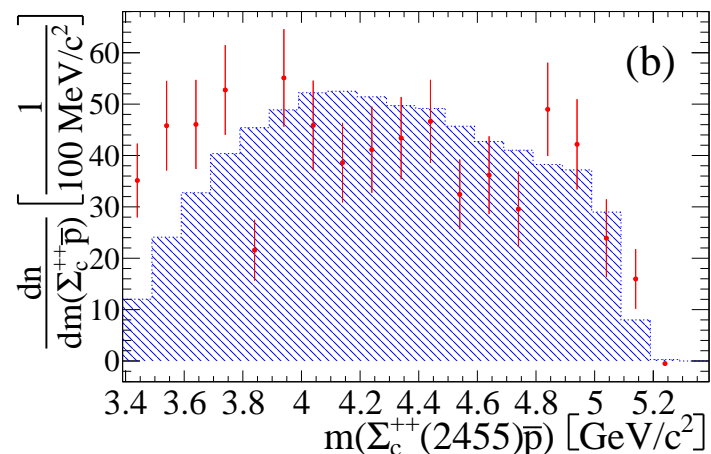

(a)

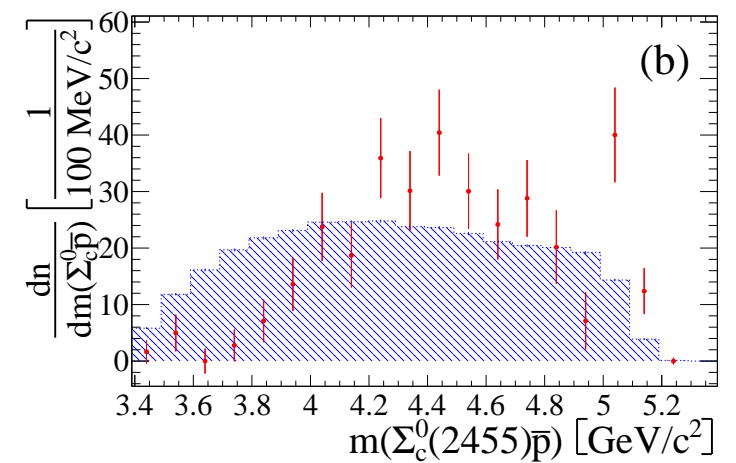

(b)

Figure 4: The invariant baryon-antibaryon mass of the decays $\bar{B}^{0} \rightarrow \Sigma_{c}^{++}(2455) \bar{p} \pi^{-}$and $\bar{B}^{0} \rightarrow$ $\Sigma_{c}^{0}(2455) \bar{p} \pi^{+}$for selected events from data (points with error bars) and from reconstructed phasespace generated MC events of the respective decay (histogram) scaled to the same integral. 
In the past years several theoretical models have been developed to describe this feature, e.g. baryon form factors, glueballs and pole models [6]. One simple ansatz to describe the threshold enhancement are QCD considerations according to which the partial decay rate $\Gamma_{i}$ is proportional to the running coupling constant $\alpha_{S}\left(q^{2}\right)$ and the gluon propagator term $1 / q^{2}$. This leads to the effect that the hadronization process prefers soft gluons and produces baryon pairs with small invariant masses.

In case of the very different behaviour of the invariant baryon-antibaryon mass distribution of $\bar{B}^{0} \rightarrow \Sigma_{c}^{++} \bar{p} \pi^{-}$and $\bar{B}^{0} \rightarrow \Sigma_{c}^{0} \bar{p} \pi^{+}$, the pole model can be used to describe this. Figure 5 shows one example diagram for both decays. The quarks that are enclosed by the dashed line are created by gluons after the $\bar{B}^{0}$ decay.

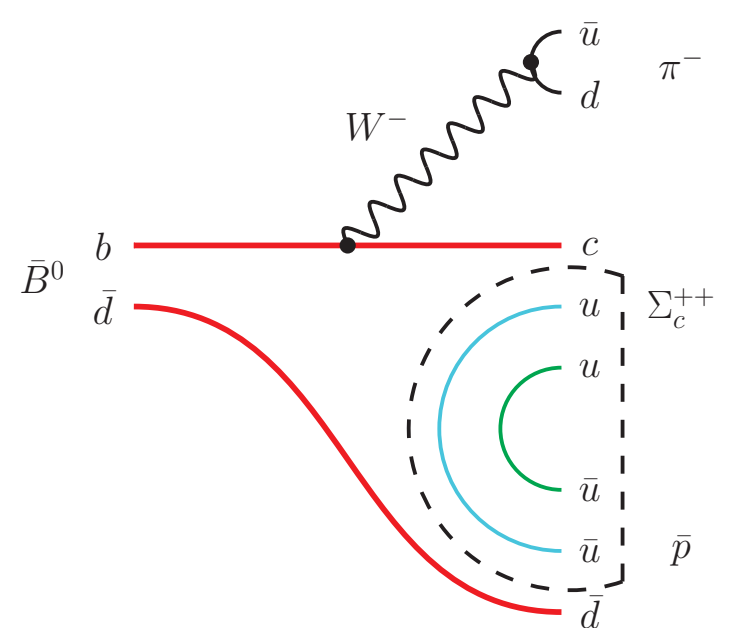

(a)

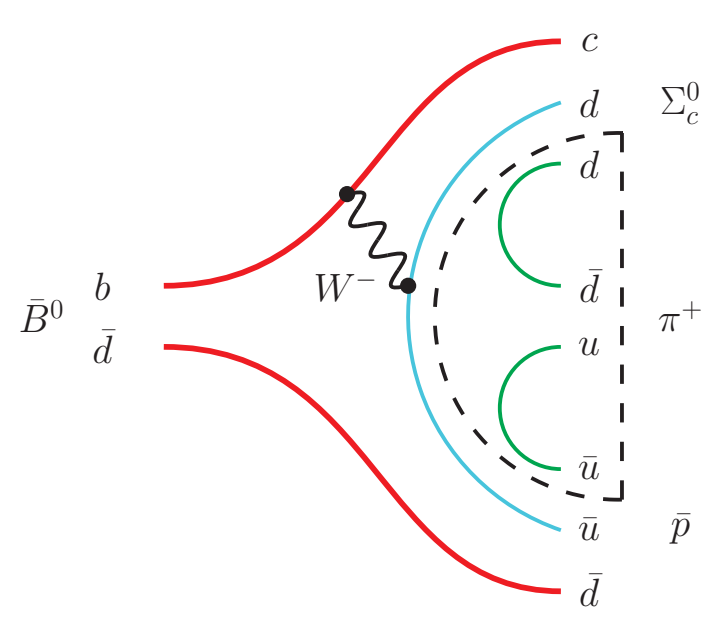

(b)

Figure 5: One example diagram for the decay $\bar{B}^{0} \rightarrow \Sigma_{c}^{++} \bar{p} \pi^{-}$and $\bar{B}^{0} \rightarrow \Sigma_{c}^{0} \bar{p} \pi^{+}$

According to the pole model the left diagram represents the decay of a $\bar{B}^{0}$ to a $\pi^{-}$and a virtual $D^{+}$ meson pole which decays via soft gluons to a $\Sigma_{c}^{++} \bar{p}$ pair with a small invariant mass. In contrast, the right diagram shows the decay of a $\bar{B}^{0}$ to a diquark pair that hadronizes to a baryon and a virtual baryon pole, but never to a meson and a virtual meson pole. In the end, the baryon pole decays to a baryon and a pion. Since the diquark content of both baryons are created back-to-back in the $\bar{B}^{0}$ rest frame, the $\Sigma_{c}^{0} \bar{p}$ pair has a high invariant mass and the extra quarks are created by hard gluons. In combination with the QCD considerations this leads to the conclusion that the matrix element of the left diagram is larger than the one of the right diagram resulting in a higher decay rate of $\bar{B}^{0} \rightarrow \Sigma_{c}^{++} \bar{p} \pi^{-}$.

In summary, the pole model and QCD considerations provide a qualitative description of the observation of an enhanced decay rate of $\bar{B}^{0} \rightarrow \Sigma_{c}^{++} \bar{p} \pi^{-}$w.r.t. $\bar{B}^{0} \rightarrow \Sigma_{c}^{0} \bar{p} \pi^{+}$and the different distributions of the invariant baryon-antibaryon mass. 


\section{6. $\bar{B}^{0} \rightarrow D^{0} \Lambda \bar{\Lambda}[7]$}

This decay can be compared to the similar decay $\bar{B}^{0} \rightarrow D^{0} p \bar{p}$ and allows studies on the $s \bar{s}$ suppression in fragmentation at low $q^{2}$ in baryonic $B$ decays. From jet fragmentation results it is predicted that $N(u \bar{u}): N(s \bar{s}) \sim 3: 1$. Under the assumption of an equal decay rate of the four possible final states $\Lambda \bar{\Lambda}, \Lambda \bar{\Sigma}^{0}, \Sigma^{0} \bar{\Lambda}$ and $\Sigma \bar{\Sigma}^{0}$ we expect that $N(p \bar{p}): N(\Lambda \bar{\Lambda}) \sim 3: \frac{1}{4}=12: 1$. We reconstruct $\Lambda \rightarrow p \pi^{-}$and $D^{0}$ in the final states $K^{-} \pi^{+}, K^{-} \pi^{+} \pi^{0}$ and $K^{-} \pi^{+} \pi^{+} \pi^{-}$. The number of signal events is determined simultaneously in an unbinned two-dimensional fit to $m\left(D^{0} \Lambda \bar{\Lambda}\right)$ and $m_{\mathrm{ES}}$ of all $D^{0}$ subsamples. In addition, contributions from $\Sigma \rightarrow \Lambda \gamma$ decays are accounted for in the fit model. Figure 6 shows the distribution of $m\left(D^{0} \Lambda \bar{\Lambda}\right)$ of the $\bar{B}^{0}$ candidates from the $D^{0} \rightarrow K^{-} \pi^{+}$subsample and the fit result.

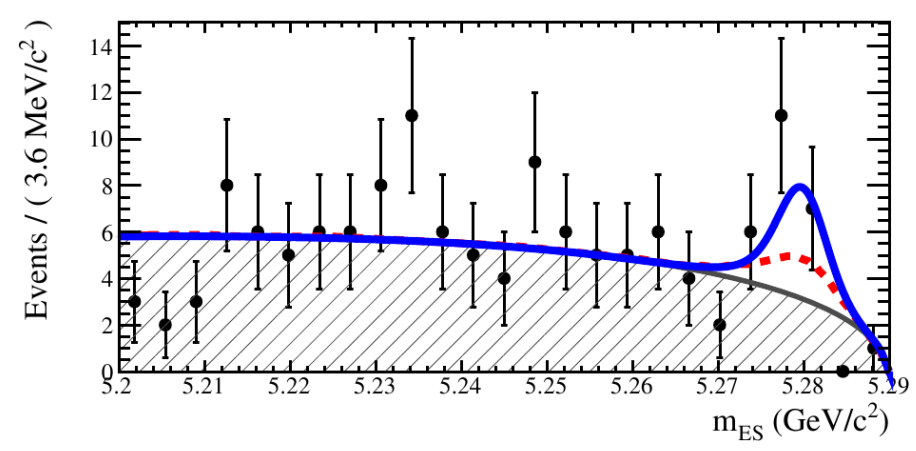

Figure 6: The distribution of $m\left(D^{0} \Lambda \bar{\Lambda}\right)$ of selected $\bar{B}^{0} \rightarrow D^{0} \Lambda \bar{\Lambda}$ candidates with $D^{0} \rightarrow K^{-} \pi^{+}$and the projection of the fit result.

The branching fraction is determined to be $\mathscr{B}\left(\bar{B}^{0} \rightarrow D^{0} \Lambda \bar{\Lambda}\right)=\left(9.8_{-2.6}^{+2.9}\right.$ stat $\left.\pm 1.9_{\text {syst }}\right) \times 10^{-6}$ corresponding to a significance of $3.4 \sigma$. In combination with the result $\mathscr{B}\left(\bar{B}^{0} \rightarrow D^{0} p \bar{p}\right)=(1.04 \pm$ $0.07) \times 10^{-4}$ [2] this leads to $\frac{\mathscr{B}\left(\bar{B}^{0} \rightarrow D^{0} p \bar{p}\right)}{\mathscr{B}\left(\bar{B}^{0} \rightarrow D^{0} \Lambda \bar{\Lambda}\right)}=10.6 \pm 3.8$, which is compatible with the aforementioned prediction of $12: 1$.

\section{References}

[1] H. Albrecht et al. [ARGUS Collaboration], Z. Phys. C 56, 1 (1992).

[2] J. Beringer et al. [Particle Data Group Collaboration], Phys. Rev. D 86, 010001 (2012).

[3] J. P. Lees et al. [BABAR Collaboration], Phys. Rev. D 87, 092004 (2013), arXiv:1302.0191 [hep-ex].

[4] J. P. Lees et al. [BABAR Collaboration], arXiv:1312.6800 [hep-ex].

[5] J. P. Lees et al. [BABAR Collaboration], Phys. Rev. D 86, 091102 (2012), arXiv:1208.3086 [hep-ex].

[6] M. Suzuki, J. Phys. G 34, 283 (2007) [hep-ph/0609133].

[7] J. P. Lees et al. [BABAR Collaboration], arXiv:1401.5990 [hep-ex]. 